LORENA CUDRIS TORRES*

Fundación Universitaria del Área Andina (Valledupar, Colombia)

ÁLVARO BARRIOS NÚÑEZ $\mathbf{N}^{* *}$

Clínica General del Caribe (Cartagena, Colombia)

\title{
Malestar psicológico en víctimas del conflicto armado ${ }^{* * *}$
}

\author{
Psychological discomfort in victims of the armed conflicto \\ Desconforto psicológico nas vítimas do conflito armado
}

\footnotetext{
* Docente Investigadora del programa de Psicología de la Fundación Universitaria del Área Andina, Líder del Grupo de Investigación Pensamiento Diverso línea Potencial Humano, salud integral y calidad de vida. Psicóloga de la Universidad Antonio Nariño, Magíster en Psicología de la Universidad Simón Bolívar, Especialista en Gerencia Pública de la Universidad de Santander, Especialista en Pedagogía para la Docencia Universitaria de la Fundación Universitaria del Área Andina, Doctorante en Ciencias de la Educación en la Universidad Cuauhtémoc de México. ORCID: https://orcid. org/oooo-00o2-3120-4757. Correo electrónico:lcudris@areandina.edu.co Reconocimientos: «Trayectoria de Excelencia 2018» categoría estudiante de doctorado-Universidad Cuauhtémoc - Septiembre de 2018; Beca Doctorado Ciencias de la Educación-Universidad Cuauhtémoc - Enero de 2017; Mención de honor por el excelente desempeño académico en la Maestría en Psicología, Universidad Simón Bolívar sede Barranquilla - Noviembre de 2016; Reconocimiento por la contribución y aporte académico, promoviendo el desarrollo humano, científico y tecnológico el beneficio de la comunidad universitaria, Universidad Franz Tamayo - Octubre de 2017; Distinción Mérito Andino - Docente Distinguido, Fundación Universitaria Del Área Andina - Mayo de 2013; Galardón 9 de Diciembre de 1983 otorgado por el Consejo Superior de la Fundación Universitaria del Área Andina, en la modalidad Gestión y Talento, por el excelente desempeño laboral durante los años 2011, 2012 y 2015 -Fundación Universitaria Del Área Andina; Mejor promedio Facultad de Psicología-Universidad Antonio Nariño abril de 2012.

** Médico de la Clínica General del Caribe, Investigador Grupo de Investigación Pensamiento Diverso. Médico Cirujano de la Universidad de Cartagena, Especialista en Gerencia en Servicios de Salud de la Universidad de Santander, Maestrando en Educación y Entornos Virtuales de Aprendizaje en la Universidad Cuauhtémoc de México. ORCID: https:// orcid.org/oooo-0o03-4153-8950. Correo electrónico: alvarobarriosn@gmail.com.

*** Revisión sistemática producto del proyecto de Investigación «Afectaciones psicológicas en víctimas del conflicto armado en el departamento del Cesar», investigación desarrollada del 15 de febrero al 30 de noviembre de 2015 con el código CV2015-Vo5 financiado por la Fundación Universitaria del Área Andina. Artículo recibido el 20-12-2017 y aprobado 16-08-2018.
} 


\section{Cómo citar}

CUdRIS TORRES, L.; BARRIOS NÚÑEZ, A. (2018).

Malestar psicológico en víctimas del conflicto armado.

Revista CS, (26), 75-90.

DOI: https://doi.org/10.18046/recs.i26.3292 
Resumen

Abstract

Resumo

La violencia, además de considerarse como una condición inherente a las relaciones humanas, también se concibe como un problema de salud pública que afecta significativamente la salud mental de las personas implicadas. De hecho, son varios los estudios que reportan las implicaciones o secuelas psicológicas en combatientes y población civil expuesta a conflictos bélicos o similares que impliquen violencia. Algunas de las cifras exponen que hasta un $30 \%$ de las personas expuestas a violencia padecen síndrome de estrés postraumático y depresión, incluso se ha reportado un 100\% en poblaciones con una afectación muy significativa (Larizgoitia et al., 2011). Por tanto, el abordaje psicológico, requiere competencias amplias que inscriban un campo de conocimiento inter o transdisciplinar que vincule las cualidades y procesos humanos, sus circunstancias vitales y contextuales en el tiempo y, particularmente, los recursos personales, familiares y comunitarios.

PALABRAS CLAVE:

Violencia; Trastorno de estrés postraumático; Depresión; Abordaje psicológico; Víctimas

Violence, in addition to being considered as an inherent condition of human relationships, is also conceived as a public health problem that significantly affects the mental health of the people involved. In fact, there are several studies that report the implications or psychological sequels in combatants and civilians exposed to war or similar conflicts that imply violence. Some of the figures show that up to $30 \%$ of people exposed to violence suffer from post-traumatic stress syndrome and depression, even 100\% have been reported in populations with a very significant impact (Larizgoitia, et al., 2011). Therefore, the psychological approach requires broad competences that inscribe an interdisciplinary or transdisciplinary field of knowledge that links human qualities and processes, their vital and contextual circumstances over time and, particularly, personal, family and community resources.

\section{KEYWORDS:}

Violence; Post-traumatic stress disorder; Depression; Psychological approach; Victims 
A violência, além de ser considerada uma condição inerente às relações humanas, também é concebida como um problema de saúde pública que afeta significativamente a saúde mental das pessoas envolvidas. De fato, há vários estudos que relatam as implicações ou sequelas psicológicas em combatentes e civis expostos à guerra ou conflitos semelhantes que implicam em violência. Alguns dos números mostram que até $30 \%$ das pessoas expostas à violência sofrem de síndrome de estresse pós-traumático e depressão, e ainda $100 \%$ foram relatados em populações com um impacto muito significativo (Larizgoitia, et al., 2011). Portanto, a abordagem psicológica requer amplas competências que inscrevem um campo de conhecimento interdisciplinar ou transdisciplinar que relaciona qualidades e processos humanos, suas circunstâncias vitais e contextuais ao longo do tempo e, particularmente, recursos pessoais, familiares e comunitários.

\section{PALAVRAS CHAVE:}

Violência; Transtorno de estresse pós-traumático; Depressão; Abordagem psicológica; Vítimas 
Este manuscrito ofrece una revisión sistemática sobre el malestar psicológico en víctimas del conflicto armado, tema que reviste importancia por el momento histórico que vive Colombia en el postconflicto y que permite, desde la academia, reflexionar sobre su abordaje.

Para la Organización Mundial de la Salud (OMS), en Colombia la presencia de síntomas emocionales y trastornos mentales entre las víctimas del conflicto es bastante alta; se ha identificado que hasta un $63 \%$ presenta algún tipo de sintomatología clínica significativa, y hasta el 33\% cumple con los criterios para el diagnóstico de un trastorno mental (Campo-Arias, Oviedo, y Herazo, 2014). Sin embargo, el conocimiento sobre la prevalencia de síntomas y presencia de trastornos mentales entre las víctimas del conflicto armado colombiano aún es escaso (Bell, Méndez, Martínez, Palma, y Bosch, 2012; Campo-Arias et al., 2014).

\section{Malestar psicológico en víctimas del conflicto armado colombiano}

Gómez Restrepo et al. (2016) describieron y contrastaron las prevalencias de algunos problemas y trastornos mentales de la población adulta en Colombia según la Encuesta Nacional de Salud Mental (ENSM, 2015), teniendo en cuenta las características del municipio de residencia respecto a su historia de violencia o conflicto armado, considerando presencia e intensidad del mismo, y describiendo a la población total y la que permaneció en la residencia. Para ello, utilizaron los resultados de los adultos (mayores de 18 años) sobre algunos problemas y trastornos mentales de la ENSM de 2015, y se clasificaron los municipios según la presencia y la intensidad del conflicto utilizando la clasificación propuesta por la CERAC. Realizaron la medición de trastorno (con el CIDI-CAPI), problemas (con AUDIT, PCL modificado) y consumo de sustancias psicoactivas.

La población entrevistada fue de 10870 personas, de las que 5429 no habían cambiado de residencia. El 21,8\% de los municipios sufrían conflicto permanente; $65,5 \%$, interrumpido; y solamente el $12,7 \%$ había sido pacificado o no tenía conflicto. La intensidad de este se reportó alta en el 31,8\%. Los municipios violentos presentaban prevalencias más altas de trastornos de ansiedad, depresivos, posible trastorno de estrés postraumático y consumo de cigarrillo. El consumo de alcohol era más frecuente en municipios con menor intensidad del conflicto.

Se encontró que la proximidad y la persistencia de eventos de conflicto armado se relacionan con una mayor prevalencia de problemas y trastornos mentales, lo cual lleva a la necesidad de planear intervenciones desde la salud pública que permitan 
mitigar el daño, tanto en situación de conflicto como tras este. La asociación entre estas variables es compleja y se entrelazan otros aspectos como el tipo de exposición, la intensidad, el tiempo ocurrido, posiblemente haberse desplazado y las características culturales que podrían influir en la adaptación y la capacidad de resiliencia de los individuos (Gómez Restrepo et al., 2016).

La situación de violencia socio política y el conflicto armado en Colombia generaron una gran cantidad de víctimas, entre las cuales se ubican personas en situación de desplazamiento, desapariciones forzadas, exposición a actos violentos, masacres y muertes violentas, entre otras circunstancias (Campo-Arias et al., 2014; Palacio, 2016).

En estudios con víctimas del conflicto armado, se han encontrado como principales impactos psicológicos: trastornos de estrés postraumático, del estado de ánimo, de ansiedad y depresión, por fobia, por consumo de alcohol con patrón no especificado, y riesgo o intento suicida (Aristizábal et al., 2012; Bell et al., 2012; Campo-Arias et al., 2014; Charry-Lozano, 2011; Gómez Restrepo et al., 2016; Hewitt Ramírez et al., 2016; Londoño et al., 2005; Ramírez-Giraldo, Hernández-Bustamante, Romero-Acosta, y Porras-Mendoza, 2017). Adicionalmente, se ha identificado disminución de los niveles de calidad de vida, ruptura de las redes sociales y afectivas, modificación de los roles familiares y desarraigo cultural (Alejo, Rueda, Ortega, y Orozco, 2007; Larizgoitia et al., 2014).

La presencia de daño psicológico, incluso cinco años después de las vivencias de las situaciones de violencia, evidencian el daño prolongado y real en términos de salud mental, teniendo en cuenta los impactos transgeneracionales de los traumas, especialmente en este tipo de vivencias (Charry-Lozano, 2011), cuestión que se agudiza según el tipo de violencia experimentada, la convivencia en el mismo contexto con los victimarios y el desplazamiento forzado (Gómez Restrepo et al., 2016; Ramírez-Giraldo et al., 2017; Morina, Rushiti, Salihu, y Ford, 2010; Steel et al., 2009; Siriwardhana, y Stewart, 2012; Jurado et al., 2017). Estos problemas de salud mental, como consecuencia de la violencia, requieren consideraciones psicosociales específicas para la formulación de planes integrales de intervención (Jaung, Jani, Banu, y Mackey, 2017; Jegannathan, Kullgren, y Deva, 2015).

Diferentes autores proponen que la perspectiva psicosocial podría plantear modelos de intervención más eficientes y acorde a los requerimientos de estas personas, los cuales orientan esta actividad hacia metas que buscan un lugar para el sujeto frente a su problemática. Esta perspectiva viabiliza cambios a nivel personal, familiar y social, y demanda el trabajo en equipos interinstitucionales e interdisciplinarios para generar un efecto consistente, considerando procesos y no acciones aisladas (Arévalo, 2010; Charry-Lozano, 2011; Estrada, Ripoll, y Rodríguez, 2010; Obando, Salcedo, y Correa, 2017; Rebolledo y Rondón, 2010). 
Otro aspecto a resaltar en las diversas intervenciones que se propongan es el papel que juega la autoestigmatización en las víctimas (Campo-Arias et al., 2014), así como la estigmatización, la cual explica el impacto de la violencia, especialmente la sexual, en la salud mental, en particular sobre los síntomas de depresión y el estrés postraumático, de tal modo que esta juega un papel importante en la conformación de las secuelas mentales de la violencia (Verelst, Schryber, Haene, Broekaert, y Derluyn, 2014).

En contextos académicos hay una investigación realizada por Rojas y Díaz (2017), quienes llevaron a cabo un estudio de caso sobre cómo acercar a los estudiantes universitarios de la Universidad Pontificia Bolivariana a los problemas del conflicto social y armado en Colombia. Mostraron la importancia de «pensar» sobre la violencia y su multiplicidad. Además, las entrevistas de víctimas sociales y de violencia armada de los estudiantes se presentan como una valiosa estrategia de sensibilización. Una comprensión más profunda de la violencia causada por el conflicto armado, junto con el contacto personal con las víctimas, permitió a los estudiantes ser más conscientes del mundo concreto y de las historias de vida que los rodean.

Como resultado del estudio, encontraron que el proceso de educación universitaria en Colombia requiere un mayor liderazgo por parte de las ciencias sociales y las humanidades. Para Rojas y Díaz (2017), una forma de lograrlo es abriendo espacios que trasciendan la rigidez de los esquemas educativos. El caso retratado es uno de los muchos que podrían contribuir a la formación contextual en la universidad, puesto que los autores piensan que el contexto es fundamental, predominantemente en las ciencias sociales y humanas, donde es crucial que los estudiantes universitarios tengan la oportunidad de abordar los fenómenos sociales y culturales y, en consecuencia, pueden contrastar las múltiples interpretaciones.

Es vital reconocer las influencias teóricas, pues las múltiples interpretaciones de la violencia son narrativas de sujetos que se han atrevido a pensar en el papel de la violencia en relación con la memoria. En Colombia no se pueden ignorar las diversas reflexiones, discusiones y aportes a la comprensión de la violencia, especialmente después de la segunda Guerra Mundial: es necesario reconocer y comenzar la ardua tarea de pensar sobre ese proceso de violencia (Rojas y Díaz, 2017).

Por tanto, es una prioridad tener referentes para el contraste, y las múltiples interpretaciones que los estudiantes abordan a través de las lecturas son el modelo para contrastar con la situación en Colombia. Un punto significativo tiene que ver con la noción de «víctima» y su relación con la transformación de la violencia en el siglo XX. Con esa referencia, los estudiantes pudieron contrastar lo que significa ser víctima en (y de) el conflicto armado colombiano (Rojas y Díaz, 2017). 
También es importante la confrontación con otras versiones sobre la violencia, después de que Rojas y Díaz (2017) recibieron en su investigación algunas aclaraciones de violencia y su contraste con la situación en Colombia, donde el punto relevante del curso era el ejercicio de investigación. Los estudiantes pudieron reunirse con las víctimas del conflicto armado colombiano y reconocieron los relatos sobre la violencia generada por la guerra. Por lo tanto, fue posible no solo reconocer la multiplicidad de la violencia, sino también la multiplicidad de narrativas que permiten la construcción de la memoria histórica.

El proceso de concientización en la educación universitaria es esencial, la conciencia hace que los estudiantes interioricen la situación de las víctimas del conflicto armado. En otras palabras, las víctimas dejan de estar fuera y lejos del proceso formativo para convertirse en sujetos narrativos que necesitan atención. Los estudiantes lo reconocieron cuando socializaron las entrevistas, y este reconocimiento confrontó a los estudiantes en un bello proceso desde fuera del aula y orientado hacia el interior de la formación: un proceso que apunta a la construcción de la memoria histórica a partir del ejercicio investigativo. Concisamente, una lucha abierta contra el olvido $\mathrm{y}$, por tanto, contra la impunidad en la historia reciente de la violencia colombiana (Rojas y Díaz, 2017).

\section{Malestar psicológico en esposas de exprisioneros de guerra y mujeres veteranas que regresan a la guerra}

Según Lahav, Stein y Salomón (2016), en su estudio denominado «Mantener una distancia saludable: auto-diferenciación y salud percibida entre las esposas de ex-prisioneros de guerra», el cautiverio de guerra puede afectar a los cónyuges de exprisioneros de guerra de muchas maneras, incluyendo los síntomas del estrés postraumático (PTSS) y las dificultades somáticas que se manifiestan en la salud percibida negativa. Esto se conoce, generalmente, como traumatización secundaria. La teoría sugiere que el desarrollo de esta se produce a través de la relación con el sobreviviente del trauma primario Figley (1986), lo que implica que la capacidad de mantener una distancia emocional equilibrada en la relación puede desempeñar un papel fundamental. No obstante, la contribución de la autodiferenciación a las perturbaciones somáticas secundarias sigue estando, en gran medida, sin investigar.

En el estudio realizado por Lahav et al. (2016), fueron evaluadas, prospectivamente, 30 (T1) y 38 (T2) años después de la Guerra del Yom Kippur de 1973, las esposas de exprisioneros de guerra $(n=143)$ y las esposas de excombatientes de control $(n=102)$. La autodiferenciación y PTSS se evaluaron en ambos puntos de tiempo, mientras que las medidas de salud percibidas fueron evaluadas en T2. 
Los autores Lahav et al. (2016) encontraron como resultado del estudio que las esposas de exprisioneros de guerra respaldaron un PTSS más alto, una autodiferenciación más baja y una salud percibida negativa, en comparación con las esposas de control. La exposición indirecta al cautiverio de guerra se relacionó con baja autodiferenciación y PTSS elevado, que predijo la salud percibida negativa. Además, la autodiferenciación caracterizada por la fusión con los demás medió completamente la relación entre la exposición indirecta al cautiverio de guerra y la salud percibida, más allá de los efectos del PTSS como mecanismo.

Los resultados sugieren que las tendencias hacia la fusión con otros dentro de la relación marital actúan como un factor de riesgo no solo para la angustia psicológica, sino también para la angustia somática entre los sobrevivientes de trauma secundario. Por lo tanto, las intervenciones clínicas pueden tratar de mejorar la capacidad de autodiferenciación de los sobrevivientes de trauma indirecto, facilitando así, potencialmente, la prevención del sufrimiento somático secundario.

Maiocco y Smith (2016) llevaron a cabo una investigación sobre la experiencia de las mujeres veteranas que regresan de la guerra, cuyo objetivo fue documentar las historias de ocho mujeres veteranas retiradas del servicio militar o activos de las reservas, que desempeñaron funciones de apoyo en Irak o Afganistán y que hablaran inglés. Se utilizó investigación cualitativa fenomenológica con la técnica entrevista, a través de la elaboración de línea de vida; las participantes expresaron quiénes eran, dónde habían estado y hacia dónde iban. Al realizar el análisis las historias fueron reconstruidas y luego compartidas con cada mujer veterana para ser confirmadas. Las veteranas afirmaron que narrar su historia tenía propiedades curativas, ya que sentían un alivio en el recuento de su experiencia.

Los resultados de este estudio muestran que la reintegración de mujeres veteranas de guerra en la comunidad es una experiencia física, emocional y espiritual que nunca termina. Las familias juegan un papel fundamental en el proceso de reinserción; sin embargo, las veteranas relataban que los amigos experimentaban diferencias entre la mujer que se había ido y la que había vuelto de la guerra. El apoyo social es un factor de protección para los veteranos en la lucha por la prevención de problemas de salud, y la ausencia de este apoyo ocasiona la presencia de alteraciones físicas, dificultades de reintegración y falta de resiliencia.

Otro dato relevante en este estudio fue el hecho de que las mujeres veteranas reportaron experimentar 2 de 3 síntomas asociados con estrés postraumático sin ningún tipo de intervención terapéutica, lo cual es preocupante, ya que las mujeres tienen mayores probabilidades de salud mental adversa que los hombres veteranos. 


\section{Método}

Se llevó a cabo una revisión de 26 artículos científicos de alto impacto, de los cuales $90 \%$ son de los últimos 5 años y $10 \%$ de la última década, a los que se accedió por medio de las siguientes bases de datos y motores de búsqueda: ResearchGate, SciELO, EBSCO, ScienceDirect, PubMed, ProQuest, Google Scholar y Scopus. Se utilizaron como palabras clave las siguientes: violencia, trastorno de estrés postraumático, depresión, abordaje psicológico, víctimas. Se diseñó una rejilla para la revisión que contempló los siguientes aspectos: palabras clave, base de datos, autor(es), año, título, revista de publicación, objetivos, variables esenciales contempladas, tipo de estudio, aspectos metodológicos relevantes y principales hallazgos y conclusiones.

\begin{tabular}{l|l} 
TABLA 1 & Textos utilizados para la revisión
\end{tabular}

\section{Nombre de la revista}

Título de artículo
Revista Colombiana de Psi- Prevalencia de síntomas, posiquiatría

bles casos y trastornos mentales en víctimas del conflicto armado en situación de desplazamiento en Colombia: Una revisión sistemática

Revista Colombiana de Psi- Estigma y salud mental en persoquiatría nas víctimas del conflicto armado interno colombiano en situación de desplazamiento forzado

Revista Colombiana de Psi- Violencia por conflicto armado quiatría y prevalencias de trastornos del afecto, ansiedad y problemas mentales en la población adulta colombiana

Journal Conflict and Health

Characteristics of the Colombian armed conflict and the mental health of civilians living in active conflict zones

Gaceta Sanitaria

Impacto de la violencia colectiva en la salud. Resultados del estudio ISAVIC en el País Vasco

Revista Colombiana de Psi- Salud mental en víctimas de quiatría
Larizgoitia, I., Izarzugaza, I., Iraurgi, I., Ballesteros, J., Forero, C., Markez, I., y Alonso, J. (2011)

Campo-Arias, A., Oviedo, H. y Herazo, E. (2014)

Campo-Arias, A. y Herazo, E. (2014).

Gómez Restrepo, C., Tamayo Martínez, N., Buitrago, G., Guarnizo, C., Garzón Orjuela, N., Eslava, J., De Vriesa, E., Rengifoh, H., Rodríguez, A., y Rincón, C. (2016).

Bell, V., Méndez, F., Martínez, C., Palma, P. P., y Bosch, M. (2012). Londoño, N. H., Muñiz, O., Correa, J. E., Patiño, C. D., Jaramillo, G., Raigoza, J., Toro, L., Restrepo, D. A., y Rojas, C. 


\begin{tabular}{|c|c|c|}
\hline Hamilton Medical & $\begin{array}{l}\text { The association between armed } \\
\text { conflict, violence and mental } \\
\text { health: a cross sectional study } \\
\text { comparing two populations in } \\
\text { Cundinamarca department, } \\
\text { Colombia }\end{array}$ & $\begin{array}{l}\text { Londoño, A., Romero, P., y Ca- } \\
\text { sas, G. (2012). }\end{array}$ \\
\hline Psicología desde el Caribe & $\begin{array}{l}\text { Síntomas y traumatismo psíqui- } \\
\text { co en víctimas y victimarios del } \\
\text { conflicto armado en el Caribe } \\
\text { colombiano }\end{array}$ & $\begin{array}{l}\text { Aristizábal, E., Palacio, J., Mada- } \\
\text { riaga, C., Osman, H., Parra, L., } \\
\text { Rodríguez, J., y López, G. (2012). }\end{array}$ \\
\hline Colombia Forense & $\begin{array}{l}\text { Impactos psicológicos y psicoso- } \\
\text { ciales en víctimas sobrevivientes } \\
\text { de masacre selectiva en el marco } \\
\text { del conflicto en el Suroccidente } \\
\text { colombiano en el año } 2011\end{array}$ & Charry-Lozano, L. (2011). \\
\hline $\begin{array}{l}\text { Revista Colombiana de Psico- } \\
\text { logía }\end{array}$ & $\begin{array}{l}\text { Afectaciones psicológicas, es- } \\
\text { trategias de afrontamiento y } \\
\text { niveles de resiliencia de adultos } \\
\text { expuestos al conflicto armado en } \\
\text { Colombia }\end{array}$ & $\begin{array}{l}\text { Hewitt Ramírez, N., Juárez, F., } \\
\text { Parada Baños, A. J., Guerrero Lu- } \\
\text { zardo, J., Romero Chávez, Y. M., } \\
\text { Salgado Castilla, A. M., y Vargas } \\
\text { Amaya, M. V. (2016). }\end{array}$ \\
\hline Psicología desde el Caribe & $\begin{array}{l}\text { Estado de salud mental de perso- } \\
\text { nas víctimas del conflicto arma- } \\
\text { do en Chengue }\end{array}$ & $\begin{array}{l}\text { Ramírez-Giraldo, A., Hernán- } \\
\text { dez-Bustamante, O., Rome- } \\
\text { ro-Acosta, K., y Porras-Mendoza, } \\
\text { E. }(2017) \text {. }\end{array}$ \\
\hline Universitas Psychologica & $\begin{array}{l}\text { Estudio epidemiológico del } \\
\text { TEPT en población desplazada } \\
\text { por la violencia política en Co- } \\
\text { lombia }\end{array}$ & $\begin{array}{l}\text { Alejo, E. G., Rueda, G., Ortega, } \\
\text { M., y Orozco, L. C. (2007). }\end{array}$ \\
\hline $\begin{array}{l}\text { Clinical Psychologica Psycho- } \\
\text { ther }\end{array}$ & $\begin{array}{l}\text { Psychopathology and well-being } \\
\text { in civilian survivors of war see- } \\
\text { king treatment: a follow-up study }\end{array}$ & $\begin{array}{l}\text { Morina, N., Rushiti, F., Salihu, } \\
\text { M., y Ford, D. (2010). }\end{array}$ \\
\hline $\begin{array}{l}\text { Journal of the American Medi- } \\
\text { cal Association }\end{array}$ & $\begin{array}{l}\text { Association of torture and other } \\
\text { potentially traumatic events } \\
\text { with mental health outcomes } \\
\text { among populations exposed to } \\
\text { mass conflict and displacement: } \\
\text { a systematic review and me- } \\
\text { ta-analysis }\end{array}$ & $\begin{array}{l}\text { Steel, Z., Chey, T., Silove, D., } \\
\text { Marnane, C., Bryant, R., y Van, } \\
\text { O. (2009). }\end{array}$ \\
\hline International Health & $\begin{array}{l}\text { Forced migration and mental } \\
\text { health: prolonged internal dis- } \\
\text { placement, return migration and } \\
\text { resilience }\end{array}$ & $\begin{array}{l}\text { Siriwardhana, C. y Stewart, R. } \\
(2012) \text {. }\end{array}$ \\
\hline $\begin{array}{l}\text { Revista de Psiquiatría y Salud } \\
\text { Mental }\end{array}$ & $\begin{array}{l}\text { Factores asociados a malestar } \\
\text { psicológico o trastornos mentales } \\
\text { comunes en poblaciones migran- } \\
\text { tes a lo largo del mundo }\end{array}$ & $\begin{array}{l}\text { Jurado, D., Alarcón, R., Martínez, } \\
\text { J., Mendieta, Y., Gutiérrez, L., y } \\
\text { Guerpegui, M. (2017). }\end{array}$ \\
\hline $\begin{array}{l}\text { Psychiatric Clinics of North } \\
\text { America }\end{array}$ & $\begin{array}{l}\text { International Emergency Psy- } \\
\text { chiatry Challenges: Disaster Me- } \\
\text { dicine, War, Human Trafficking, } \\
\text { Displaced Persons }\end{array}$ & $\begin{array}{l}\text { Jaung, M., Jani, S., Banu, S., y } \\
\text { Mackey, J. (2017). }\end{array}$ \\
\hline
\end{tabular}




\begin{tabular}{|c|c|c|}
\hline Asian Journal Psychiatric & $\begin{array}{l}\text { Mental health services in Cam- } \\
\text { bodia, challenges and opportu- } \\
\text { nities in a post-conflict setting }\end{array}$ & $\begin{array}{l}\text { Jegannathan, B., Kullgren, G., y } \\
\text { Deva, P. (2015). }\end{array}$ \\
\hline Revista de Estudios Sociales & $\begin{array}{l}\text { Atención y reparación psicoso- } \\
\text { cial en contextos de violencia so- } \\
\text { ciopolítica: una mirada reflexiva }\end{array}$ & Arévalo, L. (2010). \\
\hline Revista de Estudios Sociales & $\begin{array}{l}\text { Intervención psicosocial con fi- } \\
\text { nes de reparación con víctimas } \\
\text { y sus familias afectadas por el } \\
\text { conflicto armado interno en Co- } \\
\text { lombia: equipos psicosociales en } \\
\text { contextos jurídicos. }\end{array}$ & $\begin{array}{l}\text { Estrada, A., Ripoll, K., y Rodrí- } \\
\text { guez, D. (2010). }\end{array}$ \\
\hline Psicogente & $\begin{array}{l}\text { La atención psicosocial a perso- } \\
\text { nas víctimas del conflicto arma- } \\
\text { do en contextos institucionales } \\
\text { de salud pública }\end{array}$ & $\begin{array}{l}\text { Obando, L., Salcedo, M., y Co- } \\
\text { rrea, L. (2017). }\end{array}$ \\
\hline Revista de Estudios Sociales & $\begin{array}{l}\text { Reflexiones y aproximaciones al } \\
\text { trabajo psicosocial con víctimas } \\
\text { individuales y colectivas en el } \\
\text { marco del proceso de reparación }\end{array}$ & $\begin{array}{l}\text { Rebolledo, O. y Rondón, L. } \\
(2010) \text {. }\end{array}$ \\
\hline Child Abuse \& Neglect & $\begin{array}{l}\text { The mediating role of stigmatiza- } \\
\text { tion in the mental health of ado- } \\
\text { lescent victims of sexual violence } \\
\text { in Eastern Congo }\end{array}$ & $\begin{array}{l}\text { Verelst, A., Schryber, M., Hae- } \\
\text { ne, L., Broekaert, E., y Derluyn, } \\
\text { I. (2014). }\end{array}$ \\
\hline $\begin{array}{l}\text { Procedia-Social and Behavio- } \\
\text { ral Sciences }\end{array}$ & $\begin{array}{l}\text { Education and Sensitization on } \\
\text { Violence: Seeking to Understand } \\
\text { the Victims of the Colombian Ar- } \\
\text { med Conflict }\end{array}$ & Rojas, Y. y Díaz, S. (2017). \\
\hline $\begin{array}{l}\text { Journal of Psychosomatic Re- } \\
\text { search }\end{array}$ & $\begin{array}{l}\text { Keeping a healthy distance: } \\
\text { Self-differentiation and percei- } \\
\text { ved health among ex-prisoners- } \\
\text { of-war's wives }\end{array}$ & $\begin{array}{l}\text { Lahav, Y., Stein, J., y Zahaba, S. } \\
\text { (2016). }\end{array}$ \\
\hline $\begin{array}{l}\text { Archives of Psychiatric Nur- } \\
\text { sing }\end{array}$ & $\begin{array}{l}\text { The Experience of Women Vete- } \\
\text { rans Coming Back from War }\end{array}$ & Maiocco, G. y Smith, M. (2016). \\
\hline
\end{tabular}

\section{Conclusiones}

Las víctimas de un acontecimiento intenso amenazante tienen una mayor probabilidad de experimentar el trastorno de estrés postraumático (TEP). La literatura revisada coincide con que la violencia sociopolítica es una serie de traumas que generan con mayor frecuencia este cuadro clínico, siendo el factor central del trastorno de estrés postraumático la percepción de amenaza incontrolable por parte de la víctima a su enfermedad física o psicológica. El TEP puede estar acompañado de depresión y, en algunos casos, del consumo de sustancias psicoactivas que empeoran el pronóstico de la población. 
A partir de la información recolectada para este análisis, es posible establecer que el impacto de la violencia sobre las víctimas es evidente y significativo. Considerando las altas puntuaciones presentadas, las cuales se traducen en relevancia clínica y psiquiátrica en cada una de las investigaciones revisadas, la afectación al estado de salud mental de comunidades que han sido víctimas del conflicto armado y otro tipo de violencia requiere de atención inmediata por parte de los diferentes organismos involucrados en su proceso de recuperación.

Existen huellas de la violencia que son visibles, como las ruinas, heridas físicas, las ausencias motivadas por la muerte; pero hay otras que son invisibles y que atañen al daño moral, a los traumas psicológicos, al deterioro de los valores sobre los cuales se constituye la humanidad (Cudris, Barrios, y Jiménez, 2018). Por tanto, el abordaje psicológico requiere competencias amplias que inscriban un campo de conocimiento inter o transdisciplinar que vincule las cualidades y procesos humanos, sus circunstancias vitales y contextuales en el tiempo y, particularmente, los recursos personales, familiares y comunitarios.

Por ello, se hace necesario que el acompañamiento psicosocial a víctimas de violencia sociopolítica, desde una perspectiva de los Derechos Humanos, cuente con claridades éticas y políticas que propendan por la exigencia de sus derechos y por la construcción de estrategias de superación de la impunidad. Es difícil determinar con precisión, y en todas sus connotaciones, el daño que ha causado al país la violencia sociopolítica y el conflicto armado (Cudris et al., 2018).

Construir colaborativamente una lectura del problema y su solución, que parta de la escucha activa de la mejor manera, a fin de facilitar la participación y atención, mediante el diseño de escenarios conversacionales de convergencia. Los equipos gubernamentales hoy diseñan modelos, protocolos y rutas de atención, pero es necesario invocar la redefinición de estos de manera inter y transdisciplinar, sectorial e institucional, integrando la experiencia de afectación psicológica de las víctimas.

\section{Referencias}

ALEJO, E. G., RUEDA, G., ORTEGA, M., Y OROZCO, L. C. (2007). Estudio epidemiológico del TEPT en población desplazada por la violencia política en Colombia. Universitas Psychologica, $6,623-635$.

ARÉVALO, L. (2010). Atención y reparación psicosocial en contextos de violencia sociopolítica: una mirada reflexiva. Revista de Estudios Sociales, 36, 29-39. 
ARISTIZÁBAL, E., PALACIO, J., MADARIAGA, C., OSMAN, H., PARRA, L., RODRÍGUEZ, J., Y LÓPEZ, G. (2012). Síntomas y traumatismo psíquico en víctimas y victimarios del conflicto armado en el Caribe colombiano. Psicología desde el Caribe, 29(1), 123-152.

BELL, V., MÉNDEZ, F., MARTÍNEZ, C., PALMA, P. P., Y BOSCH, M. (2012). Characteristics of the Colombian armed conflict and the mental health of civilians living in active conflict zones. Journal Conflict and Health, 6(1), 1-8. doi:10.1186/1752-1505-6-10

CAMPO-ARIAS, A., Y HERAZO, E. (2014). Estigma y salud mental en personas víctimas del conflicto armado interno colombiano en situación de desplazamiento forzado. Revista colombiana de Psiquiatría, 43(4), 212-217. doi:http://dx.doi.org/10.1016/j.rcp.2014.09.004

CAMPO-ARIAS, A., OVIEDO, H., Y HERAZO E. (2014). Prevalencia de síntomas, posibles casos y trastornos mentales en víctimas del conflicto armado en situación de desplazamiento en Colombia: Una revisión sistemática. Revista Colombiana de Psiquiatría, 43(4), 177-185. doi:10.1016/j.rcp.2014.07.003

CHARRY-LOZANO, L. (2011). Impactos psicológicos y psicosociales en víctimas sobrevivientes de masacre selectiva en el marco del conflicto en el Suroccidente colombiano en el año 2011. Colombia Forense, 3(2), 51-6o. Recuperado de https://revistas.ucc.edu.co/index.php/ml/article/ view/1756

CUDRIS, L., BARRIOS, Á., Y JIMÉNEZ, L. (2018). Afectaciones psicologícas presentes en víctimas del conflicto armado del departamento del Cesar. En M. Silva, A. Silva, y G. Staaden (eds.), Intervenciones psicosociales cronologías contextos y realidades (pp. 121-130). Barranquilla: Universidad Metropolitana.

ESTRADA, A., RIPOLL, K., Y RODRÍGUEZ, D. (2010). Intervención psicosocial con fines de reparación con víctimas y sus familias afectadas por el conflicto armado interno en Colombia: equipos psicosociales en contextos jurídicos. Revista de Estudios Sociales, 36, 103-112. doi:10.15446/rcp.v25n1.49966

GÓMEZ RESTREPO, C., TAMAYO MARTÍNEZ, N., BUITRAGO, G., GUARNIZO, C., GARZÓN ORJUELA, N., ESLAVA, J., DE VRIESA, E., RENGIFOH, H., RODRÍGUEZ, A., Y RINCÓN, C. (2016). Violencia por conflicto armado y prevalencias de trastornos del afecto, ansiedad y problemas mentales en la población adulta colombiana. Revista Colombiana de Psiquiatría, 45(1), 147-153. doi:https://doi.org/10.1016/j.rcp.2016.11.001

HEWITT RAMÍREZ, N., JUÁREZ, F., PARADA BAÑOS, A. J., GUERRERO LUZARDO, J., ROMERO CHÁVEZ, Y. M., SALGADO CASTILLA, A. M., Y VARGAS AMAYA, M. V. (2016). Afectaciones psicológicas, estrategias de afrontamiento y niveles de resiliencia de adultos expuestos al conflicto armado en Colombia. Revista Colombiana de Psicología, 25(1), 125-140. doi:10.15446/rcp.v25n1.49966 
JAUNG, M., JANI, S., BANU, S., Y MACKEY, J. (2017). International Emergency Psychiatry Challenges: Disaster Medicine, War, Human Trafficking, Displaced Persons. Psychiatric Clinics of North America, 40(3), 565-574. doi:10.1016/j.psc.2017.05.015

JEGANNATHAN, B., KULLGREN, G., Y DEVA, P. (2015). Mental health services in Cambodia, challenges and opportunities in a post-conflict setting. Asian Journal Psychiatric, 13, 75-80. doi:10.1016/j.ajp.2014.12.006

JURADO, D., ALARCÓN, R., MARTÍNEZ, J., MENDIETA, Y., GUTIÉRREZ, L., Y GUERPEGUI, M. (2017). Factores asociados a malestar psicológico o trastornos mentales comunes en poblaciones migrantes a lo largo del mundo. Revista de Psiquiatría y Salud Mental, 10(1), 45-58. Recuperado de http://www.sciencedirect.com/science/article/pii/S1888989116300209

LAHAV, Y., STEIN, J., Y ZAHABA, S. (2016). Keeping a healthy distance: Self-differentiation and perceived health among ex-prisoners-of-war's wives. Journal of Psychosomatic Research, 89, 61-68. Recuperado de http://www.jpsychores.com/article/Soo22-3999(16)30374-9/fulltext

LARIZGOITIA, I., IZARZUGAZA, I., IRAURGI, I., BALLESTEROS, J., FORERO, C., MARKEZ, I., Y ALONSO, J. (2011). Impacto de la violencia colectiva en la salud. Resultados del estudio ISAVIC en el País Vasco. Gaceta Sanitaria, 25(2), 108-114.

LONDOÑO, N. H., MUÑIZ, O., CORREA, J. E., PATIÑO, C. D., JARAMILLO, G., RAIGOZA, J., TORO, L., RESTREPO, D. A., Y ROJAS, C. (2005). Salud mental en víctimas de la violencia armada en Bojayá (Chocó, Colombia). Revista Colombiana de Psiquiatría, 34(4), 493-505. Recuperado de http://www.redalyc.org/articulo.oa?id=80634403

LONDOÑO, A., ROMERO, P., Y CASAS, G. (2012). The association between armed conflict, violence and mental health: a cross sectional study comparing two populations in Cundinamarca department, Colombia. Hamilton Medical, 6(12), 1-10. Recuperado de https://conflictandhealth. biomedcentral.com/articles/10.1186/1752-1505-6-12

MAIOCCO, G., Y SMITH, M. (2016). The Experience of Women Veterans Coming Back from War. Archives of Psychiatric Nursing, 30, 393-399. DOI: https://doi.org/10.1016/j.apnu.2016.01.008

MORINA, N., RUSHITI, F., SALIHU, M., Y FORD, D. (2010). Psychopathology and well-being in civilian survivors of war seeking treatment: a follow-up study. Clinical Psychologica Psychother, $17,79-86$.

OBANDO, L., SALCEDO, M., Y CORREA, L. (2017). La atención psicosocial a personas víctimas del conflicto armado en contextos institucionales de salud pública. Psicogente, 2O(38), 382-397. doi:http://doi.org/10.17081/psico.20.38.2559

PALACIO, C. (2016). La salud mental y el postconflicto. Revista Colombiana de Psiquiatría, 45(4), 229. DOI: http://dx.doi.org/10.1016/j.rcp.2016.10.003 
RAMÍREZ-GIRALDO, A., HERNÁNDEZ-BUSTAMANTE, O., ROMERO-ACOSTA, K., Y PORRAS-MENDOZA, E. (2017). Estado de salud mental de personas víctimas del conflicto armado en Chengue. Psicología desde el Caribe, 34(1), 1-20.

REBOLLEDO, O., Y RONDÓN, L. (2010). Reflexiones y aproximaciones al trabajo psicosocial con víctimas individuales y colectivas en el marco del proceso de reparación. Revista de Estudios Sociales, 36, 40-50.

ROJAS, Y., Y DÍAZ, S. (2017). Education and Sensitization on Violence: Seeking to Understand the Victims of the Colombian Armed Conflict. Procedia-Social and Behavioral Sciences, 237, 562567. Recuperado de http://www.sciencedirect.com/science/article/pii/S1877042817301076

SALUD, M. D. (2015). Encuesta Nacional de Salud Mental 2015. Bogotá: MINSALUD. Recuperado de: https://www.javerianacali.edu.co/sites/ujc/files/node/field-documents/field_document_file/ saludmental_final_tomoi_color.pdf

SIRIWARDHANA, C., Y STEWART, R. (2012). Forced migration and mental health: prolonged internal displacement, return migration and resilience. International Health, 5, 19-23.

STEEL, Z., CHEY, T., SILOVE, D., MARNANE, C., BRYANT, R., Y VAN, O. (2009). Association of torture and other potentially traumatic events with mental health outcomes among populations exposed to mass conflict and displacement: a systematic review and meta-analysis. Journal of the American Medical Association, 302, 537-549.

VERELST, A., SCHRYBER, M., HAENE, L., BROEKAERT, E., Y DERLUYN, I. (2014). The mediating role of stigmatization in the mental health of adolescent victims of sexual violence in Eastern Congo. Child Abuse of Neglect, 38 (7), 1139-1146. Recuperado de http://linkinghub. elsevier.com/retrieve/pii/So145213414001343 